\title{
Dermatomiosite Juvenil e Linfoma de Hodgkin: Uma Rara Associação
}

\section{Juvenile Dermatomyositis and Hodgkin Disease: A Rare Association}

\author{
André Cavalcanti $^{(1)}$, Maria Teresa R. A. Terreri ${ }^{(2)}$, Adriana M. E. Sallum ${ }^{(3)}$, Suely K. N. Marie ${ }^{(4)}$, \\ Flávio Luisi ${ }^{(5)}$, Maria Odete E. Hilário ${ }^{(6)}$
}

\section{RESUMO}

A dermatomiosite (DM) em adultos está freqüentemente associada com câncer. Já na faixa etária pediátrica, a dermatomiosite juvenil (DMJ) é predominantemente idiopática, e sua associação com neoplasia é rara e com base apenas em relatos de casos. Embora rara, a presença de neoplasia em pacientes com DMJ deve ser sempre suspeitada quando houver manifestações clínicas atípicas e alterações laboratoriais incomuns. A seguir, descrevemos e discutimos um caso de DMJ e linfoma de Hodgkin em uma adolescente.

Palavras-chave: dermatomiosite juvenil, neoplasia, linfoma de Hodgkin, adolescência.

\section{INTRODUÇÃO}

A dermatomiosite juvenil (DMJ) é a principal miopatia inflamatória idiopática encontrada na faixa etária pediátrica. É uma doença rara caracterizada por fraqueza muscular proximal e simétrica e lesões cutâneas. Dentre as características que a diferenciam da doença do adulto estão a maior intensidade e freqüência de vasculopatia e calcinose e a rara associação com malignidade ${ }^{(1-3)}$. Pela raridade dessa associação, descrevemos e discutimos um caso de DMJ e linfoma de Hodgkin em uma adolescente.

\section{RELATO DE CASO}

Menina de 11 anos, etnia negra, encaminhada ao ambulatório de reumatologia pediátrica com queixa de pele descamativa, endurecida, e hiperemia periocular há um

\begin{abstract}
Dermatomyositis (DM) in adults is frequently associated with cancer. In contrast, during childhood juvenile dermatomyositis (JDM) is predominantly idiopathic and its association with neoplasia is rare and based only in case reports. Although rare, the presence of neoplasia in JDM patients must always be suspected in face of atypical clinical manifestations and uncommon laboratorial findings. We describe and discuss a case of JDM and Hodgkin disease in an adolescent.
\end{abstract}

Keywords: juvenile dermatomyositis, neoplasia, Hodgkin disease, adolescence.

ano que persistiam até o dia da consulta. Inicialmente a paciente foi acompanhada pelo dermatologista como uma dermatite atópica sem melhora clínica. No começo, as lesões cutâneas apresentavam uma hiperpigmentação, hiperemia e descamação em superfície extensora de joelhos, cotovelos e articulações de mãos e região de decote. Posteriormente, além da hiperpigmentação, apareceram áreas de hipopigmentação associadas a prurido. Além do quadro cutâneo, a paciente apresentava perda de $5 \mathrm{~kg}$ no período de um ano e há seis meses vinha apresentando dificuldade para caminhar e subir escadas associada à artralgia em joelhos e cotovelos. Ao exame físico, a paciente apresentava hiperemia periocular bilateral (heliotropo), face edemaciada, lesões hiperpigmentadas e hipopigmentadas com descamação em superfície extensora de joelhos, cotovelos e articulações das mãos (sinal de Gottron), face e tórax (Figura 1). Apresentava

Recebido em 02/08/07. Aprovado, após revisão, em 02/10/07. Declaramos a inexistência de conflitos de interesse.

Disciplina de Alergia, Imunologia Clínica e Reumatologia do Departamento de Pediatria da Universidade Federal de São Paulo - Escola Paulista de Medicina (Unifesp/EPM).

1. Pós-graduando da disciplina de Alergia, Imunologia Clínica e Reumatologia do Departamento de Pediatria da Unifesp/EPM.

2. Professora afiliada da disciplina de Alergia, Imunologia Clínica e Reumatologia do Departamento de Pediatria da Unifesp/EPM.

3. Professora colaboradora, doutora da Faculdade de Medicina da Universidade de São Paulo (FMUSP).

4. Professora-associada, livre-docente da FMUSP.

5. Médico do Instituto de Oncologia Pediátrica, Setor de Oncologia do Departamento de Pediatria da Unifesp/EPM.

6. Professora-associada e responsável pelo Setor de Reumatologia da disciplina de Alergia, Imunologia Clínica e Reumatologia do Departamento de Pediatria da Unifesp/EPM.

Endereço para correspondência: Maria Teresa R. A. Terreri, Rua dos Otonis, 725, CEP 04025-002, São Paulo, SP, e-mail: teterreri@terra.com.br 
também contratura em joelhos e cotovelos, força muscular grau III, sinal de Gower presente e dificuldade para elevar e sustentar a cabeça e membros inferiores. Foi palpado um linfonodo em região supraclavicular direita de $4 \mathrm{~cm}$ de diâmetro, fixo, indolor e sem sinais inflamatórios.

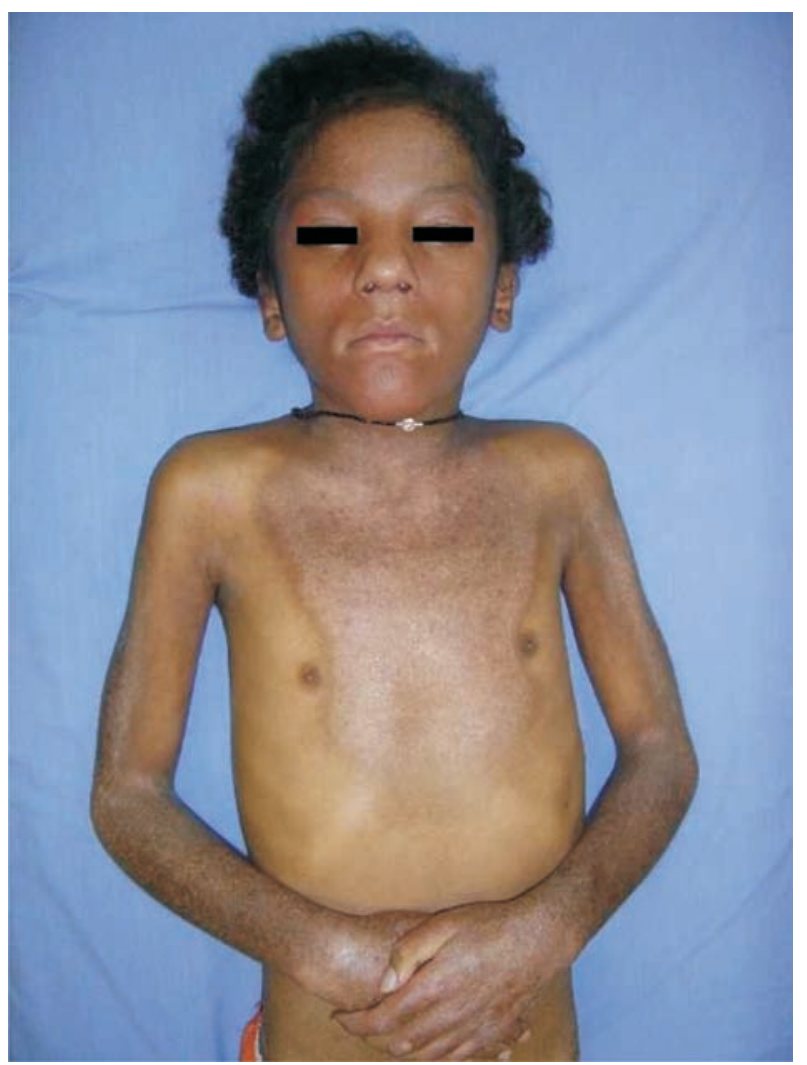

Figura 1 - Menina de 11 anos com diagnóstico de DMJ apresentando discreto heliotropo e lesões hiperpigmentadas e hipopigmentadas em face, tórax e membros.

Pelo quadro clínico da paciente, foi feito o diagnóstico de DMJ e foram solicitados exames complementares a fim de se confirmar o diagnóstico. Exames laboratoriais: hemoglobina $8,0 \mathrm{~g} / \mathrm{dL}, 13.100$ leucócitos $/ \mathrm{mm}^{3}$ com diferencial normal, 618.000 plaquetas $/ \mathrm{mm}^{3}$, velocidade de hemossedimentação (VHS) de $96 \mathrm{~mm}$ na primeira hora, proteína C reativa de 7,0 mg/dL ( normal $<0,8 \mathrm{mg} / \mathrm{dL}$ ), transaminase glutâmica oxalacética (TGO) $33 \mathrm{U} / \mathrm{L}$ (até $38 \mathrm{U} / \mathrm{L}$ ), transaminase glutâmica pirúvica (TGP) $15 \mathrm{U} / \mathrm{L}$ (até $41 \mathrm{U} / \mathrm{L})$, creatinoquinase $(\mathrm{CK}) 71 \mathrm{U} / \mathrm{L}(26-140 \mathrm{U} / \mathrm{L})$ e desidrogenase láctica (DHL) $965 \mathrm{U} / \mathrm{L}(240-480 \mathrm{U} / \mathrm{L})$. A dosagem do anticorpo antinúcleo (ANA) em células Hep-2 foi positiva com um título 1:640, padrão nuclear pontilhado fino. Os anticorpos anti-DNA e anti-ENA foram negativos. A pesquisa do anticorpo anti-tRNA-histidil sintetase (anti-Jol) também foi negativa. Anticorpo anticardiolipina IgG foi de 60 GPL e IgM 18 MPL. A biópsia de pele mostrou uma dermatite de interface com mucinose dérmica e ectasia vascular compatível com DM. A biópsia muscular mostrou intensa regeneração das fibras musculares, atrofia perifascicular e presença de infiltrado inflamatório perivascular, achados esses compatíveis com DMJ (Figura 2). A capilaroscopia periungueal mostrou intensa ectasia capilar e desvascularização de distribuição difusa compatível com o padrão SD (escleroderma). A presença de lesões cutâneas características, fraqueza muscular, aumento discreto de enzimas musculares e biópsia muscular alterada foi compatível com o diagnóstico de DMJ de acordo com os critérios de Bohan e Peter ${ }^{(4)}$.

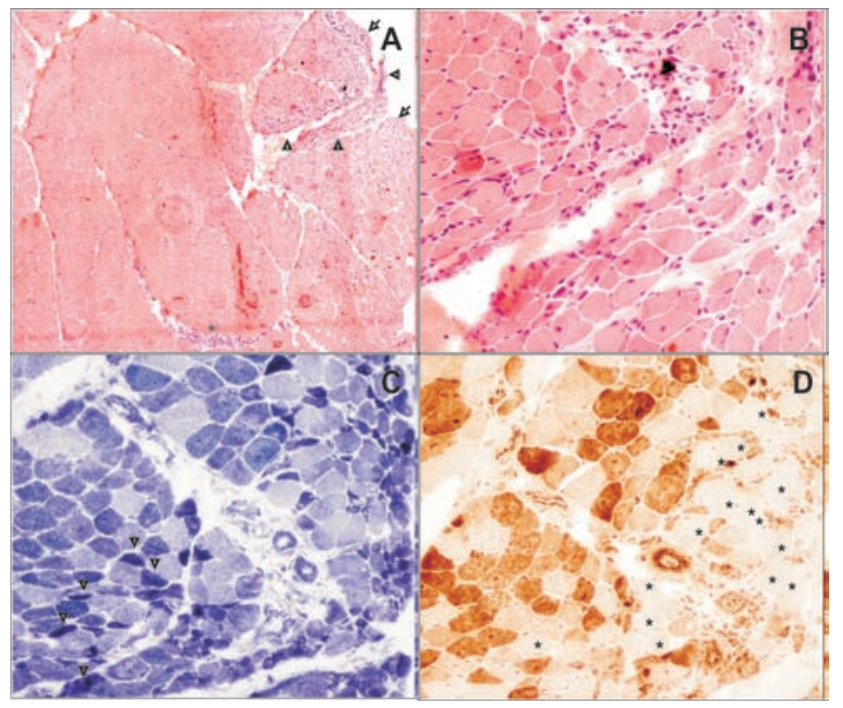

Figura 2 - Criocortes seqüenciais de biópsia muscular do músculo bíceps braquial esquerdo. A: coloração H\&E (40x) mostra a presença de atrofia perifascicular (setas) e infiltrado linfomononuclear perivascular perimisial (*). B: coloração H\&E (200X) mostra a presença de fibras de pequeno calibre, algumas com núcleo interno de distribuição na periferia do fascículo, caracterizando a atrofia perifascicular, achado característico da dermatomiosite. C: reação histoquímica oxidativa NADH (200x) mostrando a presença de fibras de pequeno calibre fortemente reativas (setas) na periferia dos fascículos e 0 desarranjo da citoarquitetura intermiofibrilar das fibras de maior calibre em disposição no interior dos fascículos. D: reação histoquímica oxidativa pela citocromo $C$ oxidase (200X) mostrando a presença de fibras com perda da atividade desta enzima $\left(^{*}\right)$.

A radiografia do tórax evidenciou alargamento em mediastino superior, médio e ântero-posterior, imagem essa confirmada pela tomografia computadorizada do tórax que mostrou uma massa paracardíaca, paratraqueal e aórtica com densidade de partes moles (Figura 3). Foi realizada biópsia excisional do linfonodo supraclavicular direito e o quadro histológico foi compatível com linfoma de Hodgkin clássico, subtipo de esclerose nodular (OMS) em sua fase celular, 

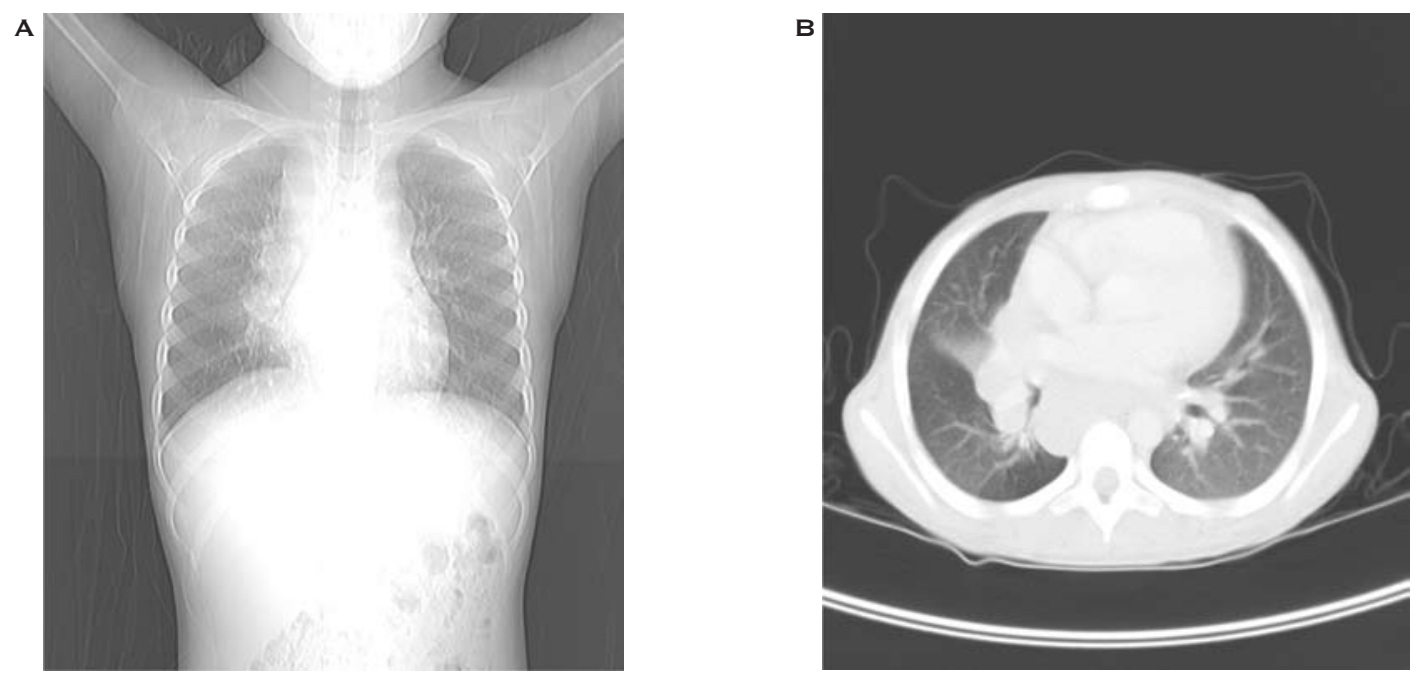

Figura 3 - Radiografia de tórax de paciente com DMJ evidenciando importante alargamento em mediastino superior, médio e ântero-posterior (A); Tomografia computadorizada de tórax revela massa paracardíaca, paratraqueal e aórtica com densidade de partes moles (B).

primário, de região cervical, com infiltração do mediastino e abdômen. Inicialmente foi optado pelo tratamento da neoplasia. A paciente foi transferida para o serviço de oncologia pediátrica; recebeu o estadiamento III B da doença de Hodgkin segundo a classificação de Ann Arbor; foi submetida ao tratamento quimioterápico-padrão: seis meses de poliquimioterapia quinzenal com ABVD (adriamicina, bleomicina, vimblastina e dacarbazina) seguida por radioterapia dos campos inicialmente envolvidos. No momento, a paciente encontra-se em remissão da doença neoplásica e fora de tratamento há um ano. Após o tratamento da neoplasia, a paciente voltou a ser acompanhada pela reumatologia pediátrica. Uma vez que não houve melhora das lesões cutâneas e da fraqueza muscular [Childhood Myositis Assessment Scale $($ CMAS $)=32$ ], foi iniciada pulsoterapia mensal com metilprednisolona $30 \mathrm{mg} / \mathrm{kg} /$ dia por três dias consecutivos e introduzido metotrexato $1 \mathrm{mg} / \mathrm{kg} / \mathrm{sem} \mathrm{VO}$.

Atualmente, a paciente apresenta melhora parcial da vasculite e da força muscular.

\section{DISCUSSÃO}

O primeiro relato de caso descrevendo a associação de malignidade (câncer de mama) e DM no adulto foi em $1916^{(5)}$. Desde então, um aumento na incidência de malignidade em pacientes com DM tem sido descrito por vários autores por intermédio de estudos populacionais (entre 6\% e $60 \%)^{(6,7)}$. Segundo alguns autores, a DM no adulto constituise num fator de risco para malignidade, o que justificaria uma triagem por exames laboratoriais e de imagem nesses pacientes, principalmente nos maiores de $50 \operatorname{anos}^{(1,7)}$. Os tipos de câncer diagnosticados nesses pacientes são similares aos mais encontrados numa determinada população de acordo com o sexo e a idade. Entre estes, estão câncer do pulmão, do ovário, da mama e do trato gastrointestinal ${ }^{(1,2,8)}$. Em contraste, a associação de neoplasias do sistema hematológico em pacientes com DM é incomum ${ }^{(6)}$.

Em pacientes adultos com DM e câncer, o diagnóstico da neoplasia foi realizado antes da DM em um terço dos casos, simultaneamente em outro terço e após o diagnóstico da DM no restante dos pacientes ${ }^{(6,8)}$. Já na série retrospectiva de Wakata et al. ${ }^{(9)}$, dos dez pacientes com DM e neoplasia, oito apresentaram o diagnóstico de câncer nos quatro primeiros anos após o diagnóstico da miopatia. Em nosso caso, o diagnóstico do linfoma de Hodgkin ocorreu simultaneamente ao diagnóstico da DMJ, embora a paciente tenha nos procurado com um ano de evolução de seus sintomas.

Alguns autores sugerem uma natureza paraneoplásica da DM em pacientes com câncer, uma vez que após o tratamento da neoplasia ocorre, geralmente, melhora da força muscular e desaparecimento das lesões cutâneas. Por outro lado, a recidiva do câncer leva a uma reativação da DM. Em nossa paciente, dois aspectos chamam a atenção: a possível relação temporal que sugeriria tratar-se de uma síndrome paraneoplásica e a não melhora da DMJ com o tratamento da neoplasia, o que pode ocorrer em alguns $\operatorname{casos}^{(8)}$. 
Recentemente, Casciola-Rosen et al. ${ }^{(10)}$ propuseram um modelo para explicar a origem paraneoplásica da miosite auto-imune em pacientes com neoplasia. O tecido muscular lesado (seja por trauma, drogas ou infecção viral) em regeneração e o tumor em questão apresentariam alta expressão de antígenos semelhantes, levando ao desenvolvimento de uma reação imunológica cruzada e ao aparecimento da miosite auto-imune.

$\mathrm{Na}$ faixa etária pediátrica, a associação entre DMJ e malignidade é extremamente rara e possui base apenas em relatos de $\operatorname{casos}^{(11-13)}$. Em três estudos com 25, 65 e 137 crianças com DMJ não foi descrito nenhum caso de associação com neoplasia ${ }^{(14-16)}$.

Até onde sabemos, existem apenas dois artigos na literatura com descrição de casos entre DMJ e linfoma de Hodgkin $^{(13,17)}$. Em um caso, o diagnóstico da miopatia precedeu o diagnóstico do linfoma e, no outro caso, o diagnóstico da DMJ e o do linfoma foram simultâneos, como ocorreu com nossa paciente. As características cutâneas e musculares nesses pacientes foram compatíveis com as da DMJ idiopática, não havendo dificuldade em seu diagnóstico. Em relação ao prognóstico, os dois pacientes entraram em remissão da neoplasia e apresentaram boa resposta clínica da DMJ com melhora da força muscular e do quadro cutâneo.

Fatores preditivos de malignidade em adultos com DMe câncer têm sido descritos por alguns autores. Acometimento cutâneo grave e extenso e VHS elevada foram os fatores mais consistentes nas diversas séries ${ }^{(1,9)}$. O nível sérico da CK como fator preditivo para malignidade é um achado controverso

\section{REFERÊNCIAS}

1. Ponyi A, Constantin T, Garami M, et al.: Cancer-associated myositis: clinical features and prognostic signs. Ann N Y Acad Sci 1051: 64-71, 2005

2. Hill CL, Zhang Y, Sigurgeirsson B, et al.: Frequency of specific cancer types in dermatomyositis and polymyositis: a populationbased study. Lancet 357(9250): 96-100, 2001.

3. Buchbinder R, Forbes A, Hall S, Dennett X, Giles G: Incidence of malignant disease in biopsy-proven inflammatory myopathy. A population-based cohort study. Ann Intern Med 134(12): $1087-95,2001$.

4. Bohan A, Peter JB: Polymyositis and dermatomyositis [part 1 of 2]. N Engl J Med 292(7): 34-7, 1975.

5. Kankeleit H: Über primäre nichteitrige Polymyositis. Dtsch Arch Klin Med 120: 335-49, 1916.

6. Maoz CR, Langevitz P, Livneh A, et al.: High incidence of malignancies in patients with dermatomyositis and polymyositis: an 11-year analysis. Semin Arthritis Rheum 27(5): 319-24, 1998. na literatura. Enquanto alguns autores sugerem que CK bastante elevada seria um fator preditivo para malignidade ${ }^{(1)}$, outros sugerem que CK normal ou levemente aumentada é que seria um fator preditivo para malignidade ${ }^{(7,9)}$.

Dourmishev ${ }^{(18)}$ descreveu diferenças entre DM idiopática e associada à neoplasia; fatores como poiquilodermia, ulcerações cutâneas, VHS elevada e doença refratária ao tratamento ou recorrente estão mais associados à DM com malignidade do que com a DM idiopática. Observamos em nossa paciente um acometimento cutâneo extenso e grave assim como uma VHS bastante elevada e discreta elevação de CK, achados esses incomuns em crianças com DMJ e descritos em adultos com DM como fatores preditores para câncer ${ }^{(1,7,9)}$. Entretanto, não existe descrição de fatores preditores na faixa etária pediátrica.

Recentemente, Targoff et al. ${ }^{(19)}$ descreveram um autoanticorpo (anti-pl55) presente em $75 \%$ dos adultos com câncer associado à $\mathrm{DM}$, sugerindo uma investigação mais detalhada para câncer nesses pacientes.

Embora seja uma associação rara na literatura, o linfoma de Hodgkin deve sempre fazer parte do diagnóstico diferencial das miopatias inflamatórias juvenis. O reumatologista pediátrico deve ficar atento para apresentações atípicas da doença, alterações laboratoriais e alguns fatores preditores para malignidade descritos em adultos. Salientamos também a importância de um exame físico minucioso, principalmente na palpação de linfonodo superficial, achado esse comum na faixa etária pediátrica, mas que em crianças com DMJ necessita de investigação detalhada, especialmente na presença de linfonodo supraclavicular.

7. Sparsa A, Liozon E, Herrmann F, et al.: Routine vs extensive malignancy search for adult dermatomyositis and polymyositis: a study of 40 patients. Arch Dermatol 138(7): 885-90, 2002.

8. Yazici Y, Kagen LJ: The association of malignancy with myositis. Curr Opin Rheumatol 12(6): 498-500, 2000.

9. Wakata N, Kurihara T, Saito E, Kinoshita M: Polymyositis and dermatomyositis associated with malignancy: a 30-year retrospective study. Int J Dermatol 41(11): 729-34, 2002.

10. Casciola-Rosen L, Nagaraju K, Plotz P, et al.: Enhanced autoantigen expression in regenerating muscle cells in idiopathic inflammatory myopathy. J Exp Med 201(4): 591-601, 2005.

11. Martini G, Calabrese F, Biscaro F, Zulian F: A child with dermatomyositis and a suspicious lymphadenopathy. J Rheumatol 32(4): 744-6, 2005.

12. Kalmanti M, Athanasiou A: Neuroblastoma occurring in a child with dermatomyositis. Am J Pediatr Hematol Oncol 7(4): 387-8, 1985.

13. Shay M, Braester A, Cohen I: Dermatomyositis as presenting symptom of Hodgkin's disease. Ann Hematol 63(2): 116-8, 1991. 
14. Shehata R, al-Mayouf S, al-Dalaan A, al-Mazaid A, al-Balaa S, Bahabri S: Juvenile dermatomyositis: clinical profile and disease course in 25 patients. Clin Exp Rheumatol 17(1): 115-8, 1999.

15. Huber AM, Lang B, LeBlanc CM, et al.: Medium- and long-term functional outcomes in a multicenter cohort of children with juvenile dermatomyositis. Arthritis Rheum 43(3): 541-9, 2000.

16. Compeyrot-Lacassagne S, Feldman BM: Inflammatory myopathies in children. Pediatr Clin North Am 52(2): 493-520, 2005.
17. Doring E, Grulich M, Dorffel W: Lymphogranulomatosis and dermatomyositis. Padiatr Grenzgeb 22(2-3): 141-7, 1983.

18. Dourmishev LA: Dermatomyositis associated with malignancy: 12 case reports. Adv Exp Med Bio 455: 193-9, 1999.

19. Targoff IN, Mamyrova G, Trieu EP, et al.: A novel autoantibody to a $155-\mathrm{kd}$ protein is associated with dermatomyositis. Arthritis Rheum 54(11): 3682-9, 2006. 